\title{
Vertical Rectus Abdominis Myocutaneous- Pedicled Island Flap for Covering Defect of the Suprapubic Area: A Case Report
}

\author{
Hyun-Dong Yeo (D), Jae-Ho Chung (D), Seung-Ha Park (D), Byung-II Lee (D), Eul-Sik Yoon (D) \\ Department of Plastic and Reconstructive Surgery, Korea University Anam Hospital, Seoul, Korea
}

\begin{abstract}
Immediate reconstruction of defects in the lower abdomen is challenging. We present a successful reconstruction case using a vertical rectus abdominis myocutaneous (VRAM) flap for a radiation ulcer on the suprapubic area following radiation therapy for recurred malignant melanoma. Before flap elevation, we conducted sufficient debridement until pliable healthy tissue was revealed. Afterwards, a $15 \times 9 \mathrm{~cm}$ VRAM flap was elevated. We then inserted the flap through a suprafascial tunnel. At 6 months postoperatively, a completely healed aesthetic flap was achieved. This case is meaningful in that it involves a successful reconstruction of a sizable abdominal wall defect of the suprapubic area with unhealthy and unviable adjacent tissue following repeated surgery and chemoradiotherapy.
\end{abstract}

Keywords: Surgical flaps; Rectus abdominis; Melanoma; Radiation ulcer

\section{Introduction}

Defects in the suprapubic region can be caused by a variety of etiologies including tumor resection, infection, and trauma. Especially in cases of refractory ulcers or large tumors in the suprapubic area, aggressive local excision is required to achieve optimal results and reduce recurrence rates. These defects require flexible and precise closure, which is challenging. Reconstruction is even more complex in cases of preoperative radiation therapy.

We present a successful reconstruction case using a vertical rectus abdominis myocutaneous (VRAM) flap for a radiation ulcer of the suprapubic area following radiation therapy for recurring malignant melanoma. This study was performed in accordance with the principles of the Declaration of Helsinki. The patient provided written informed consent for the publication and the use of her images.

\section{Case}

A 33-year-old female patient presented to our dermatology department with an enlarging dark brownish plaque measuring $2.3 \times 1.4 \mathrm{~cm}$ in the suprapubic area. Malignant melanoma was diagnosed without distant metastasis. Initially, wide excision and split-thickness skin graft were performed. The margin for free resection was confirmed by pathological results. At 2 years after the initial operation, however, a single solid nodule measuring $2.0 \times 1.7 \mathrm{~cm}$ without specific symptoms was found in the suprapubic area. Recurrence of malignant melanoma was confirmed by punch biopsy.

\section{Case Report}

Received: March 5, 2021

Revised: May 31, 2021

Accepted: June 1, 2021

\section{Corresponding author:}

Jae-Ho Chung, M.D., Ph.D.

Department of Plastic and Reconstructive Surgery, Korea University Anam Hospital, 73 Goryeodae-ro, Seongbuk-gu, Seoul 02841, Korea

Tel: +82-2-920-5368

Fax: $+82-2-920-7437$

E-mail: cjh665@gmail.com

This is an Open Access article distributed under the terms of the Creative Commons Attribution Non-Commercial License (https://creativecommons.org/licenses/by-nc/4.0/) which permits unrestricted non-commercial use, distribution, and reproduction in any medium, provided the original work is properly cited.

(c) 2021 Korean Wound Management Society 
The dermatology department performed Mohs surgery, and adjuvant chemotherapy (nivolumab) and radiation therapy were administered afterwards. A month after adjuvant concurrent chemoradiotherapy, a radiation ulcer occurred in the patient's left suprapubic area. It deteriorated further with time, displaying intensified necrosis with severe fibrosis (Fig. 1).

We considered using a pedicled flap or local flap to fill this defect. Because the patient showed compromised medical status of melanoma metastasis with a weak body status due to concurrent chemoradiotherapy, the use of a free flap, which requires lengthy surgery, was not considered. A local flap was also inapplicable because there was insufficient tissue nearby the wound. In addition, the patient weighed only $48.4 \mathrm{~kg}$ (body mass index [BMI] of $17.54 \mathrm{~kg} / \mathrm{m}^{2}$ ). In patients with lower-thanaverage BMI, we determined that a perforator flap or fasciocutaneous flap would be thin and therefore not suitable for use in a deep ulcerative wound requiring a flap with sufficient thickness. Therefore, we chose to use a pedicled VRAM flap, because it would allow for a large arc of rotation and also provide high intrinsic vascularization [1]. Preoperative computed tomography angiography was done and a right contralateral deep inferior epigastric artery was found. Consequently, we planned coverage using an inferiorly based, pedicled VRAM flap for the defect which would occur after radical debridement.

In the intraoperative view, necrosis was extended into the rectus sheath and rectus abdominis muscle. Severe fibrosis of adjacent tissue was also observed. Before flap elevation, radical debridement of devitalized tissue was performed.

After debridement, the wound bed showed a partial defect of the left rectus abdominis muscle, sized $14 \times 8 \mathrm{~cm}$. We de-
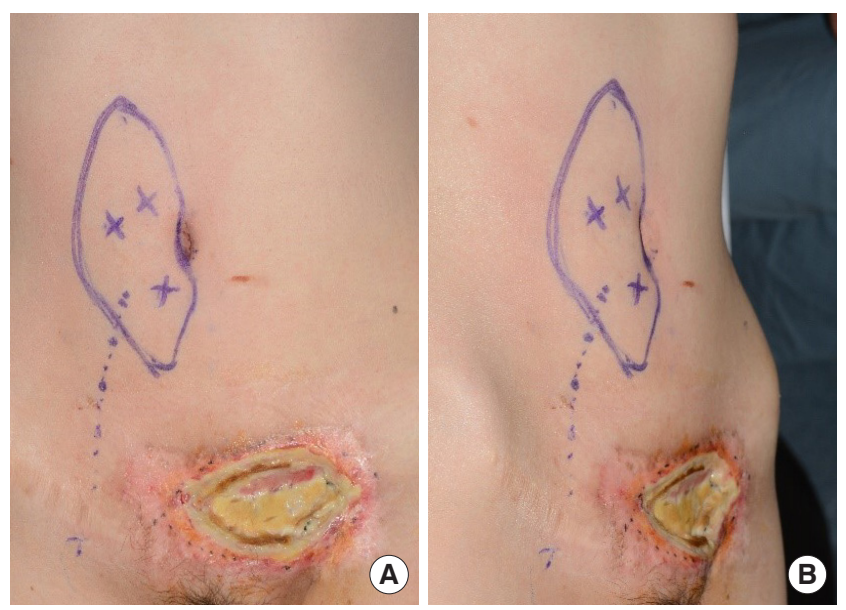

Fig. 1. Preoperative photographs of radiation ulcer at the lower abdomen. (A) Anterior view. (B) Lateral view. signed the VRAM pedicled island flap based on the right deep inferior epigastric vessels. A marked skin paddle was incised and dissected carefully. Two medial perforators and one lateral perforator were included in the flap. The rectus muscle was cut several centimeters superior to the harvested anterior rectus sheath fascia. After that, we raised the flap out of the posterior rectus sheath from superior to inferior. In order to prevent kinking and twisting of the pedicle, the attachment to the symphysis of the rectus muscle was left intact.

Eventually, a flap $15 \mathrm{~cm}$ in length and $9 \mathrm{~cm}$ in width was elevated. The flap was inset clockwise $90^{\circ}$ to reach the defect area (Fig. 2). When repairing the recipient site, the rectus abdomi-

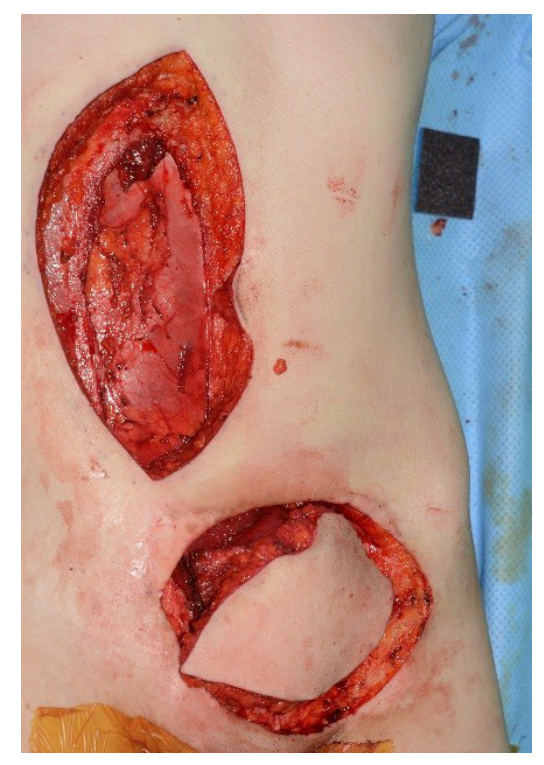

Fig. 2. Intraoperative photograph after insetting.
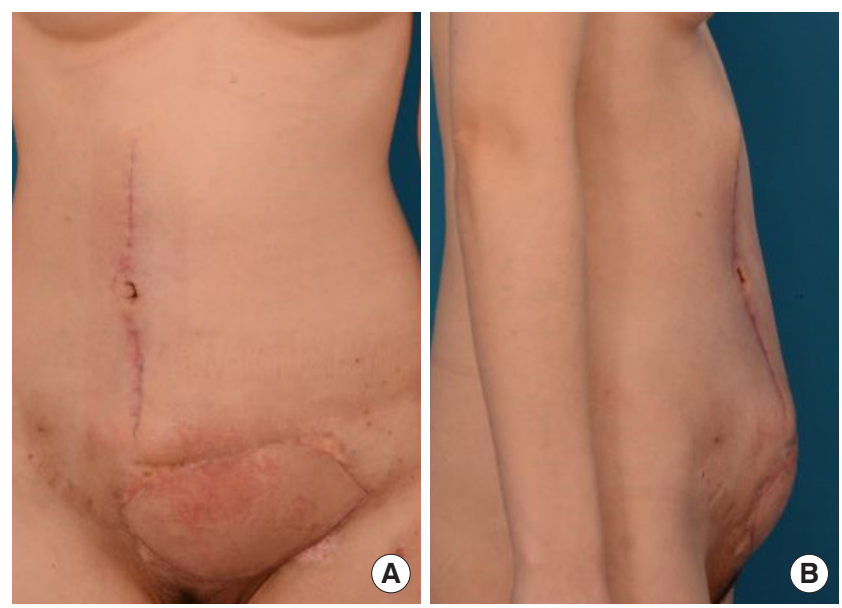

Fig. 3. Six-month postoperative photographs. (A) Anterior view. (B) Lateral view. 
nis muscle and fascia of the harvested VRAM flap were sutured to the rectus abdominis muscle and fascia, respectively, remaining on the wound bed using Vicryl 2-0. Afterwards subcutaneous suture and dermal layer suture were done. When performing closure on the donor site, the fascial defect was closed using polypropylene mesh to provide additional strength. Then the deep fat layer was sutured with Vicryl 2-0 and the dermal layer was sutured with Vicryl 3-0. At 6 months after the surgery, a completely healed aesthetic flap was achieved. Complications such as pelvic abscess, fluid collection, maceration, bulge/hernia, evisceration, flap loss secondary to ischemia and tissue necrosis, or reoperation were not observed (Fig. 3).

\section{Discussion}

There are three objectives to reconstruction of the abdominal area. The first is to restore the function and integrity of the musculofascial abdominal wall. The second is to prevent visceral eventration. The third is to provide dynamic muscle support $[2,3]$. In short, rigid functional integrity and adequate protection of abdominal cavity are required for a successful abdominal wall reconstruction. Because of these reasons, coverage of complex abdominal defects is challenging.

Treatment of abdominal defects is determined by a variety of factors, including the patient's medical condition, wound bed status and depth, and the size and location of the defects. In our case, the patient showed a compromised medical status of melanoma metastasis with a weak body status because of concurrent chemoradiotherapy. Moreover, adjacent tissue had decreased blood supply and had become fibrous due to radiation therapy, making the reconstruction more challenging [4]. Also, the radiation ulcer was made even more difficult to treat because of its location [5].

In our patient, the defect included a $13 \times 8 \mathrm{~cm}$ full-thickness skin loss of both the superficial and musculofascial layers. According to Rohrich et al. [6], a muscle flap with skin graft or musculocutaneous flap is required for cases of full-thickness defects because skin and subcutaneous tissue coverage is limited in this area. We therefore decided to use a musculocutaneous flap. The perforator flap was not considered as an option, because considering the low BMI of the patient, the flap would be thin.

Hurwitz and Hollins [7] have classified abdominal wall defects into six subunits based on horizontal and vertical planes, providing a systematic approach to reconstruction. The loca- tions were then subdivided into (1) midline and lateral, and (2) upper, middle, and lower third of abdomen. Based on Hurwitz and Hollins's classification, Rohrich et al. [6] have proposed an algorithm for reconstructing an abdominal wall defect. According to their algorithm, for full-thickness defects on the lower third of the abdomen, a rectus abdominis muscle flap, tensor fascia lata flap, rectus femoris flap, and gracilis flap can be used. It was therefore appropriate to use a vertical rectus abdominis muscle flap in our patient.

The rectus abdominis flap has a few advantages. First, the rectus abdominis flap is thick, making it possible to obliterate dead space and reduce the risk of fluid collection. Second, this flap has a large arc of rotation and high intrinsic vascularization that can improve blood supply to the recipient area [1].

In this case, there were no postoperative complications. However, the lower abdomen, which is the recipient site, appears bulkier due to the addition of the harvested rectus abdominis muscle of VRAM flap to the rectus abdominis muscle that was left on the wound bed. We did not use mesh to repair the recipient site because it was possible to suture the rectus abdominis muscle and fascia of the harvested VRAM flap to the rectus abdominis muscle and fascia, respectively, remaining on the wound bed.

The overall aesthetic outcome was satisfying, but umbilical distortion to the right side was observed. In abdominally based flap reconstruction, creating a natural-looking umbilicus during donor-site closure is important for patient satisfaction. In donor site closure, a combination of umbilical transposition would have created a more natural and aesthetically pleasing umbilicus, which would have resulted in better cosmetic results.

This case is meaningful in that it involves a successful reconstruction for a sizable abdominal wall defect of the suprapubic area with unhealthy and unviable adjacent tissues due to repetitive surgery and chemoradiotherapy. This report provides guidance for cases in which previous chemoradiotherapy has left a large abdominal wall defect with a poor wound bed.

\section{Conflict of interest}

No potential conflict of interest relevant to this article was reported.

\section{ORCID iDs}

Hyun-Dong Yeo https://orcid.org/0000-0002-5499-5053 
Jae-Ho Chung https://orcid.org/0000-0002-8351-2444

Seung-Ha Park https://orcid.org/0000-0002-2667-2791

Byung-Il Lee

https://orcid.org/0000-0001-8183-0388

Eul-Sik Yoon

\section{References}

1. Coombs DM, Patel NB, Zeiderman MR, et al. The vertical rectus abdominis musculocutaneous flap as a versatile and viable option for perineal reconstruction. Eplasty 2017;17:ic2.

2. Core GB, Grotting JC. Reoperative surgery of the abdominal wall. In: Grotting JC, editor. Aesthetic and reconstructive plastic surgery, vol. 2. St. Louis: Quality Medical Publishing Inc.; 1995. p. 1327-75.

3. DiBello JN Jr, Moore JH Jr. Sliding myofascial flap of the

rectus abdominus muscles for the closure of recurrent ventral hernias. Plast Reconstr Surg 1996;98:464-9.

4. Haubner F, Ohmann E, Pohl F, et al. Wound healing after radiation therapy: review of the literature. Radiat Oncol 2012; 7:162.

5. Porshinsky B, Ramasastry S. Abdominal wall reconstruction with free flaps. Clin Plast Surg 2006;33:269-80.

6. Rohrich RJ, Lowe JB, Hackney FL, et al. An algorithm for abdominal wall reconstruction. Plast Reconstr Surg 2000; 105:202-16.

7. Hurwitz DJ, Hollins RR. Reconstruction of the abdominal wall and groin. In: Cohen M, editor. Mastery of plastic and reconstructive surgery, vol. 2. 1st ed. Boston: Little, Brown; 1994. p. 1349-59. 